\title{
AÇÕES DE NUTRIÇÃO NO PROJETO BANDEIRA CIENTÍFICA: ESTUDO DAS EXPEDIÇÕES DE 2005 A 2010
}

\author{
ACTIONS OF NUTRITION IN "BANDEIRA CIENTÍFICA" PROJECT: \\ STUDY OF THE EXPEDITIONS FROM 2005 TO 2010
}

*Jaqueline Lopes Pereira, **Viviane Laudelino Vieira, ***Luiz Fernando Ferraz da Silva, *****Patricia Constante Jaime

\section{RESUMO}

Bandeira Científica é um projeto de extensão universitária da Universidade de São Paulo, que consiste em expedições para cidades brasileiras com baixo Índice de Desenvolvimento Humano, a fim de melhorar as condições de saúde da população. Diversas áreas integram o Bandeira Científica, dentre elas, a Nutrição. Este artigo visa descrever e avaliar a participação da equipe de Nutrição no projeto Bandeira Científica. Foi realizada pesquisa do tipo avaliativa, desenvolvida por meio de análise de relatórios de cada expedição e entrevistas com ex-participantes da equipe de Nutrição do Bandeira Científica. As atividades realizadas em cada expedição em que houve participação da Nutrição (2005 a 2010) são descritas e comparadas com as ações preconizadas pelo Ministério da Saúde. A participação da equipe de Nutrição vem se ampliando e estabilizando no Bandeira Científica. As experiências obtidas a cada ano de participação no projeto permitiram que as ações de Nutrição no Bandeira Científica buscassem, de maneira convergente ao preconizado pelo Ministério da Saúde, melhorar a saúde da população das cidades em que atua e desenvolver os participantes do projeto tanto no âmbito profissional quanto humano.

Palavras-chave: Extensão universitária. Nutrição. Atenção básica em saúde.

\section{ABSTRACT}

Bandeira Científica is a university extension project of São Paulo University, which consists in expeditions to Brazilian cities with low Human Development Index, intending to improve health conditions in the cities visited. Several undergraduate courses take part in the Bandeira Gientífica project, among them, Nutrition. This article aims to describe and evaluate the participation of the Nutrition team in the Bandeira Científica project. It was made an evaluative research, using documentary analysis and interviews with former members of Nutrition team in Bandeira Científica project. The nutrition activities developed in the expeditions from 2005 until 2010 were described and compared to the food and nutrition actions in primary care preconized by Brazilian Ministry of Health. The participation of Nutrition team has been amplifying and settling. The experiences accumulated during the project expeditions converge with the preconized food and nutrition actions on primary care, resulting in activities that might improve the health condition of the population of the visited cities. These experiences also help to develop professional and human skills and competencies among the students who are members of the project.

Key words: University extension. Nutrition. Basic health care.

\footnotetext{
* Graduanda em Nutrição da Faculdade de Saúde Pública da Universidade de São Paulo (FSP-USP) e participante do projeto Bandeira Científica - e-mail: jaquelinelopes@usp.br. ** Nutricionista, doutoranda em Nutrição em Saúde Pública da FSP-USP e discutidora do Projeto Bandeira Científica. *** Médico, professor doutor da Faculdade de Medicina da Universidade de São Paulo (FM-USP) e coordenador do projeto Bandeira Científica. **** Nutricionista, professora doutora da FSP-USP e coordenadora geral de Alimentação e Nutrição da CGAN.
} 


\section{INTRODUÇÃO}

Griado em 1957 pela Faculdade de Medicina da Universidade de São Paulo (FMUSP) e coordenado pelo Departamento de Patologia da FMUSP, o Bandeira Científica $(\mathrm{BC})$ é um projeto de extensão universitária que consiste em expedições para cidades brasileiras com baixo Índice de Desenvolvimento Humano (IDH), envolvendo, atualmente, acadêmicos de múltiplas unidades da Universidade de São Paulo, tais como Medicina, Fisioterapia, Nutrição, Fonoaudiologia, Engenharia Givil e Ambiental, Audiovisual, entre outras.

Segundo informações oficiais do projeto [I], no período de 1957 a I969, os focos eram a pesquisa científica e a educação. Devido a problemas políticos que ocorriam no Brasil nessa época, o projeto precisou ser interrompido e foi retomado apenas em I998. Desde então, o BC se propõe a alinhar atividades educacionais, científicas e assistenciais às necessidades das cidades que visita, procurando cumprir seu papel de projeto universitário, com atuação efetiva na formação dos alunos de graduação, não apenas focalizando preceitos técnicos, mas também valorizando o desenvolvimento da cidadania ao futuro profissional.

O projeto busca atuar de forma a garantir o desenvolvimento da saúde das regiões em que atua, compreendido como o "completo estado de bem-estar bio-psico-social” [I4], que inclui não apenas a abordagem das doenças, mas também dos aspectos ambientais e sociais a elas relacionadas. Devido a esse objetivo, foram incorporadas outras áreas além da Medicina, iniciando pela Fisioterapia, em 2002; a Nutrição, por sua vez, aderiu ao BC em 2005. Atualmente, diversos outros cursos integram o projeto, tais como Psicologia, Odontologia, Fonoaudiologia, Engenharia e Agronomia.

Com o objetivo de melhorar as condições de saúde da população da cidade visitada, percebeu-se que as emergentes e crescentes demandas de atenção à saúde decorrem, principalmente, dos agravos que acompanham as doenças crônicas não transmissíveis (DCNT) e as deficiências nutricionais, ambas associadas a uma alimentação e a modos de vida não saudáveis [5].

Vários estudos mostram que o Brasil convive com a transição nutricional, determinada frequentemente pela má alimentação [2, 9]. Algumas de suas consequências, as DGNT, são associadas às causas de morte mais comuns atualmente. Segundo a Organização Mundial da Saúde, a hipertensão arterial e a obesidade correspondem aos dois principais fatores de risco responsáveis pela maioria das mortes e doenças no mundo [I5]. No Brasil, as doenças cardiovasculares correspondem à primeira causa de morte há mais de 40 anos, acompanhada de um aumento expressivo da mortalidade por diabetes [7]. Além disso, esse crescimento da obesidade e suas complicações vêm ocorrendo de maneira significativa também na população de baixa renda do país [8].

Nesse contexto, a instituição da Política Nacional de Promoção da Saúde pelo Ministério da Saúde em 2006, com o objetivo de promover a qualidade de vida e reduzir a vulnerabilidade e os riscos à saúde relacionados aos seus determinantes e condicionantes, tem como um dos seus eixos estratégicos a promoção da alimentação saudável [4].

De acordo com Burlandy, citado por Vasconcelos (2008), constatam-se distintas possibilidades de intervenção em nutrição no âmbito da saúde coletiva, sendo a atenção básica em saúde, representada, sobretudo, pelo Programa de Saúde da Família (PSF), um espaço privilegiado para se avançar na promoção da alimentação saudável e na conquista da Segurança Alimentar e Nutricional (SAN), para a qual a integralidade e a intersetorialidade representam princípios fundamentais [I2].

Portanto, as ações de alimentação e nutrição na atenção básica tanto contribuirão para a qualificação como para a garantia da integralidade da atenção à saúde prestada à população [5]. Por essas razões, este trabalho tem como objetivo descrever e avaliar a participação da equipe de Nutrição no projeto de extensão universitária Bandeira Científica.

\section{MATERIAIS E MÉTODOS}

O presente trabalho consiste em pesquisa do tipo avaliativa, desenvolvido de agosto de 2010 a julho de 2011 por meio de análise de relatórios de cada expedição, disponíveis na página eletrônica do projeto, e em entrevistas com ex-participantes da equipe de Nutrição do BC.

Foram entrevistados 20 participantes, alguns presentes em mais de uma expedição. Foram obtidos dados de I4 alunos que fizeram parte do projeto durante a graduação, denominados bandeirantes, 7 alunos da graduação que desempenhavam, prioritariamente 
funções de gestão do projeto, nomeados diretores e de II nutricionistas, cujo papel era o de supervisionar as atividades desenvolvidas pelos alunos, chamados discutidores. A "Tabela I" (ver Anexo) descreve o número de participantes entrevistados e as cidades em que ocorreu o projeto desde o início da participação da Nutrição.

Os membros da equipe de Nutrição foram convidados a participar da pesquisa via $e$-mail e aqueles que aceitaram foram entrevistados pessoalmente. Foram realizadas entrevistas semiestruturadas, abordando os seguintes temas: caracterização do participante do projeto; preparação, treinamentos, atividades e características da expedição a qual participou e opiniões sobre o projeto e a participação da nutrição. As entrevistas foram gravadas e, posteriormente, transcritas.

Para realização deste trabalho, houve análise e aprovação do Comitê de Ética em Pesquisa da Faculdade de Saúde Pública da Universidade de São Paulo, pelo Protocolo de Pesquisa n. 2.I6I.

\section{RESULTADOS}

Em todos os anos, é realizado um complexo processo de preparo, com treinamentos e extenso planejamento da viagem. Sempre ocorre uma pré-visita, na qual alguns diretores vão até a cidade meses antes da expedição para averiguar as necessidades da mesma, e a infraestrutura necessária para que seja possível receber o projeto. $\mathrm{O}$ auge do $\mathrm{BC}$ ocorre durante a expedição, que é o período em que todos os integrantes vão para a cidade escolhida e realizam as atividades planejadas, durante cerca de dez dias. Aproximadamente seis meses após a expedição, alguns diretores retornam à cidade a fim de expor os resultados obtidos com os dados coletados e propor debates e soluções entre representantes da cidade, gestores e profissionais de saúde.

Porém, apesar de possuir uma estrutura previamente organizada, cada expedição tem características próprias; as atividades se adaptam às peculiaridades de cada município e, também, às dos participantes, que são responsáveis por elaborar tudo o que será realizado. A "Tabela 2" (ver Anexo) apresenta a caracterização geral de cada expedição, e a "Tabela 3" (ver Anexo), uma síntese das atividades realizadas pela equipe de Nutrição nesse período.

Em todas as expedições foram realizados treinamentos técnicos para preparar os estudantes de
Nutrição no desenvolvimento das atividades propostas, como padronização em antropometria, aulas de dietoterapia e conduta em atendimento, entre outros, de acordo com as características de cada grupo e com o tipo de atividade.

Quanto ao planejamento e preparo das atividades, em 2005, este foi organizado pela nutricionista responsável pela pesquisa realizada. Nos anos posteriores, os diretores coordenavam o grupo de bandeirantes que, em conjunto, determinavam e elaboravam as ações a serem feitas na cidade, sob orientação dos discutidores.

Outra característica comum a todos os anos em que a Nutrição participou foi o levantamento de dados epidemiológicos e nutricionais, para possibilitar o conhecimento da população atendida, subsidiando relatórios fornecidos aos gestores da cidade, com propostas de soluções para os principais problemas encontrados.

\section{EXPEDIÇÃO DE 2005, JOÃO CÂMARA (RN)}

Esta expedição apresentou um único objetivo: coletar dados para realização de pesquisa científica que, por sua vez, visava à avaliação do estado nutricional dos moradores das diversas casas visitadas pelo projeto por meio de questionários e antropometria (peso e estatura). Tais informações subsidiaram, posteriormente, orientações nutricionais de acordo com a realidade e as possibilidades de cada região ou sub-região do município. Participaram da expedição 5 acadêmicos da Faculdade de Saúde Pública da Universidade de São Paulo (FSP-USP) e 4 da Universidade Federal do Rio Grande do Norte (UFRN), faculdade local convidada a participar, além de I nutricionista que supervisionava o trabalho dos estudantes. Com os dados coletados nessa pesquisa, foi produzido um artigo científico [II], como mostra a "Tabela 4" (ver Anexo).

\section{EXPEDIÇÃO DE 2006, MACHADINHO D'OESTE (RR)}

Este foi o primeiro ano que a Nutrição incorporou a figura do diretor. A equipe foi formada por 7 acadêmicos da FSP-USP, 5 de uma instituição de ensino local (Faculdade São Lucas) e I discutidora. A partir desse ano, os objetivos da Nutrição no PBC começaram a mudar.

O foco principal da expedição de 2006 foram os atendimentos nutricionais individualizados (ANI), que ocorreram em postos de atendimento improvisados em escolas, para indivíduos com distúrbios ou 
doenças cujo controle dietético contribui ao tratamento, com avaliação nutricional seguida de orientações práticas em relação aos alimentos e dietas.

Também ocorreram atividades de capacitação junto aos agentes comunitários de saúde (ACS), com temas como Higiene e Guidados com Alimentos, Dietoterapia e Utilização do Sistema de Vigilância Alimentar e Nutricional (SISVAN). A escolha dos temas, em todos os anos, sempre é pautada pela alta prevalência das doenças e/ou suas complicações na comunidade, e pelo importante papel que os ACS desempenham no controle e prevenção destas afecções. O objetivo dessa atividade é consolidar conceitos sobre esses assuntos, compreender o contexto em que os ACS estão inseridos e construir, em conjunto, estratégias de ação.

Além das atividades planejadas, durante a expedição, surgiram oportunidades para atuar em outras atividades, tais como a participação nas Visitas Domiciliares (VD), em que os participantes do projeto formavam equipes que atendiam indivíduos com algum tipo de dificuldade de locomoção em sua própria casa, e em uma atividade multidisciplinar num assentamento do Movimento dos Trabalhadores Sem Terra (MST), na qual bandeirantes de Nutrição informavam a população local sobre como ter uma alimentação saudável. Outra atividade não planejada consistiu no desenvolvimento de oficinas de alimentação saudável para aqueles que se encontravam na fila de espera dos postos de atendimento. Essa ação surgiu a partir de observação pelos alunos da Nutrição das pessoas que permaneciam por longos períodos esperando pelo atendimento, sem participar de nenhuma atividade, e viram uma oportunidade de atuar nessa situação.

\section{EXPEDIÇÃO DE 2007, PENALVA (MA)}

As experiências de 2006 tornaram possível o desenvolvimento de outras atividades para a expedição de 2007. Houve ampliação da equipe, com 8 graduandos da FSP-USP, 4 nutricionistas de São Paulo, 7 bandeirantes e I nutricionista da universidade local convidada, a Faculdade Santa Terezinha (CEST). Também houve ampliação das ações em Nutrição.

Dentre as atividades planejadas, destaca-se o atendimento nutricional em grupo (ANG) destinado a indivíduos com características semelhantes, que podem debater suas dúvidas e encontrar, em conjunto, soluções para problemas em comum. Porém, esta não ocorreu devido à dificuldade em agrupar estas pessoas nos postos de atendimento, dado que muitas não podiam esperar por muito tempo para serem atendidos.

Outras atividades que merecem destaque são a oficina de alimentação saudável e aproveitamento integral de alimentos com merendeiras nas escolas, com elaboração de receitas para ilustrar o conteúdo; e o desenvolvimento do projeto Horta na Escola em conjunto com a equipe da Escola Superior de Agricultura "Luiz de Queiroz" da USP (ESALQ), cujo objetivo era construir hortas em espaços nas escolas a fim de reduzir o custo da merenda escolar e estimular o consumo de frutas, verduras e legumes dos alunos e suas famílias.

Também se perspectivou o levantamento de dados sobre as condições higiênico-sanitárias dos distribuidores de alimentos da cidade (por exemplo, abatedouro e mercado local) a fim de avaliar riscos e sugerir melhorias para esses estabelecimentos, mas a ação foi inviabilizada por questões políticas e de aceitação dos proprietários dos locais a serem visitados.

Este foi o primeiro ano em que a Nutrição realizou a administração das refeições para todos os integrantes do projeto, associada ao treinamento de higiene e manipulação de alimentos para as merendeiras responsáveis por preparar essas refeições.

Por outro lado, durante a expedição, surgiram oportunidades de realizar outras atividades. A antropometria da população atendida nos postos, realizada até aquele momento pelos alunos de Medicina, contou com o apoio da equipe de Nutrição. Também se desenvolveram atividades educativas com pescadores da região, que foram orientados sobre alimentação saudável e modo de preparo dos alimentos, e com professores, sobre a importância da alimentação adequada para as crianças.

\section{EXPEDIÇÃO DE 2008, ITAOBIM (MG)}

A equipe foi formada por 6 acadêmicos da FSPUSP, 4 da universidade local convidada (Universidade Federal de Minas Gerais), 3 nutricionistas de São Paulo e outra atuante na rede pública do município visitado. Diversas atividades que foram bem sucedidas no ano anterior se repetiram neste ano, porém adaptadas às especificidades da região. A antropometria realizada nos postos de atendimento tornou-se atividade exclusiva da equipe de Nutrição. Surgiu, também, uma nova atividade: a oficina educativa com crianças, que ocorria nas escolas, em conjunto com a Odontologia, nas quais foram trabalhadas a questão da alimentação 
saudável e prevenção de cáries com este público.

Todas as atividades propostas nesse ano ocorreram; porém, algumas delas tiveram que ser reduzidas em número de atuações, tais como as ações educativas em escolas, devido à indisponibilidade do próprio município.

Outra atividade prejudicada foi a VD, devido à ausência do paciente a ser visitado no momento em que a equipe chega a sua residência.

\section{EXPEDIÇÃO DE 2009, IVINHEMA (MS)}

A expedição contou com II graduandos em Nutrição da FSP-USP, I da universidade local convidada, a Universidade para o Desenvolvimento do Estado e da Região do Pantanal (Uniderp), e 5 discutidores. Dentre as atividades planejadas, foi proposta consultoria para uma cooperativa da cidade que produzia doces caseiros, para elaboração de rótulos dos produtos, avaliação das condições higiênico-sanitárias do local de preparo e treinamento de higiene e manipulação de alimentos para os responsáveis pela elaboração dos doces. A consultoria para a cooperativa de doces não ocorreu, pois os dados solicitados no período anterior à expedição - como, por exemplo, as receitas elaboradas - não foram fornecidos, e o representante da cooperativa com quem era feito contato parou de responder às solicitações da equipe do projeto BC.

Também se planejou e desenvolveu atividade com pedagogas com o tema de alimentação e o desenvolvimento neuropsicomotor de crianças, em conjunto com a Fonoaudiologia, com a proposta de esclarecer dúvidas sobre o tema e ressaltar a importância da alimentação adequada para o desenvolvimento infantil.

Foi planejada uma pesquisa com os beneficiários do Bolsa Família, para avaliação do cumprimento das condicionalidades do Programa, mas não se concretizou por dificuldades de encaminhamento da população para entrevista nos postos de atendimento.

Mais uma vez, surgiram novas atividades para o período da expedição: uma atividade com pais na Pastoral da Griança e uma gincana com crianças sobre alimentação num evento que ocorria na cidade no mesmo período.

Dados coletados nessa expedição geraram um trabalho [I3], apresentado no Congresso Mundial de Nutrição e Saúde Pública de 20 Io (ver Anexo, Tabela 4).

\section{EXPEDIÇÃO DE 2010, INHAMBUPE (BA)}

Participaram I3 acadêmicos da FSP-USP, 2 da Universidade Federal da Bahia (UFBA), 3 nutricionistas da USP e I da UFBA. Novamente, algumas atividades de anos anteriores foram realizadas, de acordo com a realidade do município. Além destas, uma nova proposta de reuniões interdisciplinares ao longo do ano, anteriormente à expedição, possibilitou a realização de diversas atividades com determinados grupos populacionais, com participação de diferentes áreas, enriquecendo os debates e a visão de cada uma delas.

Ocorreram atividades sobre saúde com jovens, gestante e nutrizes, idosos, professores, entre outros, e a participação da Nutrição, em conjunto com a Fisioterapia, num grupo de atendimento a hipertensos que já existia na cidade, o Hiperdia. A Fonoaudiologia se integrou à oficina com crianças - que ocorria somente com a Odontologia e a Nutrição - e agregou novos conhecimentos a essa atividade.

$\mathrm{Na}$ atividade com os professores, o objetivo do encontro foi conhecer o cotidiano de trabalho dos professores da rede municipal, os desafios enfrentados e as soluções encontradas. Foram apresentadas técnicas que podem ser utilizadas como atividades didáticas, para familiarizar as crianças sobre a importância de consumo de alimentos com alto valor nutricional.

Com os jovens, foram abordados temas considerados importantes a partir de questões que emergiram no momento da pré-visita, tais como os seus anseios, projetos de vida e suas perspectivas profissionais, o corpo e a sexualidade, o consumo de álcool e drogas, alimentação e as relações familiares e de amizade.

$\mathrm{Na}$ atividade direcionada a gestantes e nutrizes, as áreas participantes sortearam diversas perguntas com temas centrais do pré-natal e puerpério, com a finalidade de promover uma discussão sobre o tema, com trocas de experiências e resolução de possíveis dúvidas.

Com os ACS foram abordados temas de dietoterapia na hipertensão, diabetes, dislipidemias e tabagismo, e também o tema de saneamento básico e higiene de alimentos.

Ocorreram atividades em conjunto com a Engenharia sobre utilização do hipoclorito de sódio e higienização de alimentos para homens e mulheres responsáveis pelo tratamento da água da família e também para o público infantil, com o foco em educação ambiental associada à higiene pessoal e de alimentos.

Em 20IO, a proposta de ANG foi executada 
com sucesso, sendo que nos postos de atendimento foi possível reunir pessoas com características em comum e realizar o atendimento. Nesses momentos, os indivíduos podem compartilhar dificuldades e encontrar, em conjunto, soluções para problemas semelhantes.

Além disso, a nutrição foi novamente responsável pela administração das refeições e treinamento de manipulação de alimentos com os funcionários que as preparavam, que eram, em sua maioria, merendeiras das escolas. Devido à demanda dos mesmos, no último dia da expedição foi realizada uma atividade com todos esses funcionários sobre alimentação saudável.

A experiência dessa expedição possibilitou a elaboração de um trabalho apresentado no X Congresso de Nutrição e Alimentação [ıO], conforme descrito na "Tabela 4" (ver Anexo).

\section{DISCUSSÃO}

A atual situação epidemiológica brasileira, representada pelo aumento da incidência de doenças crônicas não transmissíveis e ainda com a presença relevante de deficiências nutricionais, justifica a importância de alunos e profissionais da área da Nutrição em uma equipe multidisciplinar que busca atender às necessidades em saúde da população do município visitado de maneira integral. Compete ao nutricionista, dentre suas atribuições na área de Saúde Coletiva, prestar assistência e desenvolver atividades educativas a coletividades ou indivíduos por meio de ações, programas, pesquisas e eventos, direta ou indiretamente relacionados à alimentação e nutrição, visando à prevenção de doenças, promoção, manutenção e recuperação da saúde [3]. Com base nestes preceitos, a equipe de Nutrição vem buscando aprimorar sua atuação a cada ano que participa do projeto $\mathrm{BC}$, contribuindo para o desenvolvimento de ações integradas às demais áreas, tal como expõe a Matriz de Ações de Alimentação e Nutrição na Atenção Básica de Saúde do Ministério da Saúde [5], que propõe que as ações do nutricionista ocorram em conjunto com equipe multiprofissional, com profissionais com conhecimentos, habilidades e atitudes que possibilitem o trabalho em equipe, competências compartilhadas e humanização no atendimento.

As ações de alimentação e nutrição no projeto têm ocorrido de maneira convergente e complementar às demais atividades que vêm sendo realizadas nos municípios visitados, procurando, por meio de comunicação com gestores e nutricionistas, atender às necessidades do município sem ultrapassar as barreiras existentes entre um projeto de extensão universitária e o governo de cada cidade. Esse é um dos motivos pelo qual, algumas vezes, nem todas as atividades planejadas no período anterior à expedição são executadas.

É possível notar que as atividades propostas pela Nutrição no BC buscam se aproximar ao máximo do preconizado na atenção básica em saúde, visto que se sabe que a baixa oferta de ações primárias de alimentação e nutrição na rede de unidades de saúde, ou sua baixa incorporação pelas equipes de saúde, implica em limitar o cumprimento dos princípios da integralidade, universalidade e resolubilidade de atenção à saúde [5].

Atualmente, vem sendo sistematizadas e organizadas as ações de alimentação e nutrição e o cuidado nutricional de acordo com os sujeitos das ações [5]. $\mathrm{O} \mathrm{BC}$, neste contexto, apresenta algumas atividades com o foco no indivíduo, como os ANI, outras, a família, como as $\mathrm{VD}$, e também, a comunidade, como nas ações educativas nas escolas, para fomentar hábitos alimentares adequados. Assim, as atividades conseguem trabalhar assuntos importantes com todos os sujeitos, utilizando uma abordagem adequada para cada um.

Ao comparar os níveis de intervenção propostos pela Matriz [5] com o projeto, nota-se que as atividades da BC buscam englobar todos eles. O diagnóstico, importante para caracterização da população a ser estudada, é realizado por meio da coleta de variáveis socioeconômicas, culturais e de saúde e avaliação nutricional no período da expedição e apresentado no momento da pós-visita, gerando discussões e propostas de ação para gestores e representantes da população da cidade, que possibilita desenvolvimento de ações no nível de gestão das ações de alimentação e nutrição. A promoção da saúde e a prevenção de doenças e distúrbios nutricionais são abordadas na realização de ações educativas para diversos públicos, com dinâmica e linguagem específicas para cada um. Já a assistência, tratamento ou cuidado ocorrem em atendimentos individuais, em grupo ou na VD, mediante as necessidades de cada indivíduo.

Quanto ao caráter das ações, vislumbrado como universais e específicas [5], a realização de atividades educativas em espaços comunitários contemplam a primeira característica, enquanto que os grupos de atendimento para gestantes e nutrizes atendem à segunda. 
Apesar de ter conseguido englobar ações tão amplas, dificuldades para a execução do projeto são encontradas a cada ano. Porém, estas vêm sendo contornadas por meio de discussão e avaliação de cada expedição, contribuindo para o amadurecimento das ações planejadas.

Um ponto crítico para a realização do trabalho é a comunicação com a cidade onde ocorre a expedição. Algumas atividades tiveram que ser remanejadas ou, até mesmo, não puderam ocorrer devido à dificuldade de contato com a população, o que ocasionou falta de público, como, por exemplo, a atividade com os cuidadores na expedição de 20IO. Esse é um processo difícil, que demanda tempo para que haja sensibilização das lideranças comunitárias para direcionamento da população que possa se beneficiar das ações que ocorrerão durante a expedição.

Outra questão complexa do projeto é o trabalho interdisciplinar. No início da participação da Nutrição no projeto $\mathrm{BC}$, ele ocorria de maneira pontual. Entretanto, com o decorrer dos anos e o amadurecimento dos novos cursos que passaram a integrar o projeto, a interdisciplinaridade passou a ser uma questão muito trabalhada e valorizada, pois se percebeu a riqueza de olhares diferentes sobre uma mesma situação. As discussões geradas possibilitam que alunos e profissionais aprendam ainda mais e os resultados obtidos sejam melhores [6]. O papel da diretoria do BC nessa questão é de essencial importância, ao facilitar e estabelecer comunicação entre pessoas de diferentes cursos no período anterior à expedição e propor que trabalhem em conjunto; porém, a visão de cada participante do projeto interfere no resultado final. É interessante observar que, no início da expedição, o trabalho conjunto é mais difícil; contudo, com o passar dos dias, a interdisciplinaridade ocorre de maneira mais natural, pois as pessoas passam a se conhecer melhor e têm maior facilidade para se comunicar.

Também é importante que exista preocupação com a continuidade do projeto depois do final de cada expedição. Por esse motivo, o BC propõe ações para que as atividades realizadas no período em que o projeto está na cidade não se percam, como, por exemplo: o encaminhamento adequado dos pacientes atendidos ao serviço de saúde da cidade; a capacitação de multiplicadores, como as crianças, os professores e os ACS; a parceria com a Universidade local, para que possa existir um acompanhamento mais próximo e a utilização de tecnologia, como a Telemedicina, possibilitando a troca de informações e discussão de casos. Dessa maneira, é possível que ações realizadas durante o período em que o projeto permaneceu na cidade se perpetuem e não desapareçam.

\section{CONCLUSÕES}

A participação da equipe de Nutrição vem se ampliando e estabilizando no projeto BC. As experiências obtidas a cada ano de participação no projeto permitiram que as ações de nutrição no BC buscassem, de maneira convergente ao preconizado pelo Ministério da Saúde, melhorar a saúde da população das cidades em que atua e desenvolver os alunos e profissionais participantes do projeto tanto no âmbito profissional quanto humano.

Porém, é importante que exista um arquivo que detalhe as experiências da Nutrição em cada ano de atuação, pois como a diretoria muda a cada expedição, apesar de fornecer os conhecimentos necessários para seus sucessores, dados muito anteriores podem acabar se perdendo e deixando de enriquecer as expedições seguintes.

\section{REFERÊNCIAS BIBLIOGRÁFICAS}

[I] BANDEIRA CIENTÍFICA. Desenvolvido pelo projeto Bandeira Científica da Universidade de São Paulo. Apresenta o histórico, as atividades e parcerias do projeto Bandeira Científica. Disponível em: <http://www. bandeiracientifica.com.br $>$. Acesso em: 5 jul. $201 \mathrm{I}$.

[2] BATISTA FILHO, M.; RISSIN, A. A transição nutricional no Brasil: tendências regionais e temporais. Gadernos de Saúde Pública, Rio de Janeiro, v. I9, supl. I, p. Si8I-Sigi, 2003. Disponível em: <http:// www.scielo.br/pdf/\%०D/csp/vigsi/aIgvigsi.pdf $>$. Acesso em: 6 jul. $201 \mathrm{II}$.

[3] BRASIL. Conselho Federal de Nutricionistas. Resolução $n^{\circ} 380 / 2005$. Dispõe sobre a definição das áreas de atuação do nutricionista e suas atribuições, estabelece parâmetros numéricos de referência, por área de atuação, e dá outras providências. Brasília, DF, 2005, 45 p.

[4] BRASIL. Ministério da Saúde. Secretaria de Vigilância em Saúde. Secretaria de Atenção à Saúde. Política nacional de promoção da saúde. Brasília: Ministério da Saúde, 2006, 60 p. Série B. Textos Básicos de Saúde.

[5] BRASIL. Ministério da Saúde. Secretaria de Atenção 
à Saúde. Departamento de Atenção Básica. Matriz de ações de alimentação e nutrição na atenção básica de saúde. Brasília: Ministério da Saúde, 2009, 78 p. Série A. Normas e Manuais Técnicos.

[6] GOMES, R.; DESLANDES, S. F. Interdisciplinaridade na saúde pública: um campo em construção. Revista Latino-Americana de Enfermagem, Ribeirão Preto, v. 2, n. 2, jul. I994. Disponível em: <http://www. scielo.br/scielo.php?script=sci_arttext\&pid=SOIO4II69I994000200008\&lng=pt\&nrm=iso >. Acesso em: I3 jul. $20 \mathrm{II}$.

[7] LESSA, I. Doenças crônicas não transmissíveis no Brasil: um desafio para a complexa tarefa da vigilância. Ciência \& Saúde Coletiva, Rio de Janeiro, v. 9, n. 4, p. 93I-943, out.-dez. 2004. Disponível em: <http://www. scielo.br/scielo.php?script $=$ sci_arttext\&pid $=$ SI 4 I 3 8I2320040004000I4\&lng=en\&nrm=iso >. Acesso em: 6 jul. $20 I I$.

[8] MONTEIRO, G. A.; CONDE, W. L. A. Tendência secular da obesidade segundo estratos sociais: Nordeste e Sudeste do Brasil, I975-I989-I997. Arquivos Brasileiros de Endocrinologia \& Metabologia, São Paulo, v. 43, n. 3, junho I999. Disponível em: 〈http://www. scielo.br/scielo.php?script=sci_arttext\&pid=SoO०4 $27301999000300004 \& \operatorname{lng}=\mathrm{en} \& \mathrm{nrm}=\mathrm{iso}>$.Acesso em: I4 jul. $201 \mathrm{I}$

[9] MONTEIRO, C. A. et al. Da desnutrição para a obesidade: A transição nutricional no Brasil. In: MONTEIRO, G. A. (Org.). Velhos e novos males da saúde no Brasil, 2. ed. São Paulo: Editora Hucitec, 2000, p. 247-255.

[Io] PEREIRA, J. L.; SILVA, G. M.; GIUDICI, K. V. Projeto de extensão interdisciplinar como parte da formação pessoal e profissional de estudantes de Nutrição em universidade pública brasileira. In: X CONGRESSO DE NUTRIÇÃO E ALIMENTAÇÃO \& II CONGRESSO IBERO-AMERICANO DE NUTRIÇÃO, 2OII, Lisboa. Anais do X Congresso de Nutrição e Alimentação \& II Congresso Ibero-Americano de Nutrição. Lisboa: Associação Portuguesa de Nutricionistas, 20II. Não paginado.

[iI] SALDIVA, S. R. D. M.; SILVA, L. F. F.; SALDIVA, P. H. N. Avaliação antropométrica e consumo alimentar em crianças menores de cinco anos residentes em um município da região do semiárido nordestino com cobertura parcial do programa bolsa família. Revista de Nutrição, Campinas, v. 23, n. I, p. 2II-229, mar.-abr. 2010.

[i2] VASCONCElos, A. G. G. P.; PEREIRA, I. D. F.; GRUZ, P J. S. C. Práticas educativas em nutrição na atenção básica em saúde: reflexões a partir de uma experiência de extensão popular em João Pessoa - Paraíba. Revista de APS, Juiz de Fora, v. II, n. 3, p. 334-340, jul.-set. 2008. Disponível em: <http://www.aps.ufjf.br/index.php/aps/article/ view/347/I25/>. Acesso em: I2 jul. 201 I.

[I3] VIEIRA, V. L. et al. Health profile of the Family Grant Program (FGP) beneficiaries in the Municipality of Ivinhema, Mato Grosso do Sul, Brazil. In: II WORLD CONGRESS OF PUBLIC HEALTH NUTRITION, 2OIO, Porto. Abstracts of II World Congress of Public Health Nutrition. Porto: Scientific Sponsorship, 2010. v. I3. p. 53.

[I4] WHO - World Health Organization. Constitution of the World Health Organization. Basic Documents. Geneva: World Health Organization, I946.

[I5] WHO - World Health Organization. The World Health Report 2002: reducing risks, promoting healthy life. Geneva: World Health Organization, 2002.

\section{AGRADECIMENTOS}

À Pró-Reitoria de Cultura e Extensão Universitária pelo apoio financeiro à bolsista e aos patrocinadores do projeto Bandeira Científica. 


\section{ANEXO}

TABELA 1

Número de ex-participantes da equipe de Nutrição do projeto BC entrevistados, de acordo com a expedição. São Paulo, 201 I.

\begin{tabular}{|c|c|c|c|c|c|}
\hline ANO & CIDADE DA EXPEDIÇÃO & BANDEIRANTES & DIRETORES & DISCUTIDORES & TOTAL \\
\hline 2005 & $\begin{array}{l}\text { João Câmara, Jandaíra } \\
\text { e Bento Fernandes (RN) }\end{array}$ & I & o & 0 & I \\
\hline 2006 & Machadinho D'Oeste (RO) & 3 & I & I & 5 \\
\hline 2007 & Penalva (MA) & 3 & I & 2 & 6 \\
\hline 2008 & Itaobim (MG) & 3 & 2 & 3 & 8 \\
\hline 2009 & Ivinhema (MS) & 4 & 2 & 5 & II \\
\hline \multirow[t]{2}{*}{2010} & Inhambupe (BA) & O & I & O & I \\
\hline & Total & I4 & 7 & II & 32 \\
\hline
\end{tabular}

TABELA 2

Número de acadêmicos participantes e de atendimentos no projeto BC no período de 2005 a 2010 . São Paulo, $201 \mathrm{I}$.

\begin{tabular}{lccccccc}
\hline & 2005 & 2006 & 2007 & 2008 & 2009 & 2010 & MÉDIA \\
\hline Estudantes participantes (Todas as áreas) & II8 & I43 & 236 & I54 & I66 & I75 & I65 \\
\hline Estudantes de Nutrição Participantes & 9 & I2 & I5 & IO & I2 & I5 & I2 \\
\hline Atendimentos Totais (Todas as áreas) & 3.659 & 4.425 & 4.592 & 3.935 & 3.302 & 4.304 & 4.036 \\
\hline Atendimentos Individuais de Nutrição & 504 & 359 & 458 & 284 & $4 \mathrm{I} 3$ & $33 \mathrm{I}$ & 392 \\
\hline Visitas Domiciliares & - & I3 & 35 & 26 & 28 & 22 & 25 \\
\hline
\end{tabular}


TABELA 3

Síntese das atividades planejadas e realizadas pela equipe de Nutrição no período de 2005 a 2010. São Paulo, 201 I.

\begin{tabular}{|c|c|c|}
\hline $\begin{array}{l}\text { CIDADE E ANO } \\
\text { DA EXPEDIÇÃO }\end{array}$ & ATIVIDADES PLANEJADAS & ATIVIDADES REALIZADAS \\
\hline $\begin{array}{c}\text { João Câmara, Jandaíra } \\
\text { e Bento Fernandes } \\
\text { (RN) / } 2005\end{array}$ & $\begin{array}{c}\text { Antropometria } \\
\text { Avaliação Nutricional de Grianças }\end{array}$ & $\begin{array}{c}\text { Antropometria } \\
\text { Avaliação Nutricional de Crianças }\end{array}$ \\
\hline $\begin{array}{l}\text { Machadinho D'Oeste } \\
\text { (RO) / } 2006\end{array}$ & $\begin{array}{c}\text { ANI } \\
\text { Gapacitação de ACS }\end{array}$ & $\begin{array}{c}\text { ANI } \\
\text { Capacitação de ACS } \\
\text { Orientação Nutricional em assentamento } \\
\text { VD } \\
\text { Oficina em postos de atendimento }\end{array}$ \\
\hline $\begin{array}{c}\text { Penalva } \\
(\mathrm{MA}) / 2007\end{array}$ & $\begin{array}{c}\text { ANI e ANG } \\
\text { VD } \\
\text { Capacitação de ACS } \\
\text { Oficina com Merendeiras } \\
\text { Horta nas Escolas } \\
\text { Consultoria Sanitária } \\
\text { Investigação sobre anemia } \\
\text { Administração de refeições }\end{array}$ & $\begin{array}{c}\text { Antropometria } \\
\text { ANI } \\
\text { VD } \\
\text { Capacitação de ACS } \\
\text { Oficina com Merendeiras } \\
\text { Horta nas Escolas } \\
\text { Administração de refeições } \\
\text { Orientação Nutricional com Pescadores } \\
\text { Oficina com Professores }\end{array}$ \\
\hline $\begin{array}{c}\text { Itaobim } \\
(\mathrm{MG}) / 2008\end{array}$ & $\begin{array}{c}\text { Antropometria } \\
\text { ANI } \\
\text { VD } \\
\text { Capacitação de ACS } \\
\text { Oficina com Merendeiras e Pais } \\
\text { Horta nas Escolas } \\
\text { Educação Nutricional com Crianças } \\
\text { Administração de refeições }\end{array}$ & $\begin{array}{c}\text { Antropometria } \\
\text { ANI } \\
\text { VD } \\
\text { Capacitação de ACS } \\
\text { Oficina com Merendeiras e Pais } \\
\text { Horta nas Escolas } \\
\text { Educação Nutricional com Grianças } \\
\text { Administração de Refeições }\end{array}$ \\
\hline $\begin{array}{l}\text { Ivinhema } \\
(\mathrm{MS}) / 2009\end{array}$ & $\begin{array}{c}\text { Antropometria } \\
\text { ANI e ANG } \\
\text { VD } \\
\text { Capacitação de ACS } \\
\text { Educação Nutricional com Crianças } \\
\text { Consultoria para desenvolvimento de rótulos } \\
\text { para doces caseiros } \\
\text { Orientação Nutricional com Pedagogas } \\
\text { Avaliação Nutricional de Escolares } \\
\text { Pesquisa PBF } \\
\text { Administração de refeições }\end{array}$ & $\begin{array}{c}\text { Antropometria } \\
\text { ANI } \\
\text { VD } \\
\text { Capacitação de ACS } \\
\text { Educação Nutricional com Crianças } \\
\text { Orientação Nutricional com Pedagogas } \\
\text { Administração de refeições } \\
\text { Atividade Pastoral da Criança } \\
\text { Atividades lúdicas sobre Nutrição com } \\
\text { Crianças }\end{array}$ \\
\hline
\end{tabular}




\section{Antropometria \\ ANI e ANG \\ VD}

Atividade com ACS

Inhambupe

(BA) / 2010
Educação Nutricional com Crianças

Orientações sobre saúde com jovens, professo-

res, gestantes/nutrizes, idosos e cuidadores

Higiene de alimentos com Crianças e Adultos

Orientação Nutricional com Hipertensos

Composteira

Administração de refeições
Antropometria

ANI e ANG

VD

Atividade com ACS

Educação Nutricional com Grianças

Orientações sobre saúde com jovens, pro-

fessores, gestantes/nutrizes e idosos

Higiene de alimentos com Crianças e Adultos

Orientação Nutricional com Hipertensos

Capacitação com Merendeiras

Administração de refeições

ACS: Agentes Comunitários de Saúde

ANG: Atendimento Nutricional em Grupo

ANI: Atendimento Nutricional Individual

PBF: Programa Bolsa Família

VD: Visita Domiciliar

TABELA 4

Produção Científica da equipe de Nutrição do projeto BC, de acordo com a expedição. São Paulo, 20 II.

\begin{tabular}{|c|c|c|c|}
\hline $\begin{array}{l}\text { CIDADE DA } \\
\text { EXPEDIÇÃOO }\end{array}$ & $\begin{array}{c}\text { TIPO DE } \\
\text { PRODUÇÃO }\end{array}$ & TíTULO & PUBLICAÇÃO \\
\hline $\begin{array}{c}\text { João Câmara, Jandaíra } \\
\text { e Bento Fernandes } \\
\text { (RN) }\end{array}$ & Artigo Científico & $\begin{array}{l}\text { Avaliação antropométrica e consumo } \\
\text { alimentar em crianças menores de cinco } \\
\text { anos residentes em um município da re- } \\
\text { gião do semiárido nordestino com cober- } \\
\text { tura parcial do programa bolsa família. }\end{array}$ & $\begin{array}{c}\text { Rev. Nutr., Campinas, } \\
\text { 23(2):2II-229, mar./abr., } \\
2010\end{array}$ \\
\hline Ivinhema (MS) & $\begin{array}{c}\text { Apresentação em } \\
\text { Ciongresso }\end{array}$ & $\begin{array}{c}\text { Health profile of the Family Grant } \\
\text { Program (FGP) beneficiaries in the } \\
\text { Municipality of Ivinhema, Mato Grosso } \\
\text { do Sul, Brazil. }\end{array}$ & $\begin{array}{c}\text { II World Congress of Public } \\
\text { Health Nutrition, 2OIO, } \\
\text { Porto. }\end{array}$ \\
\hline Inhambupe (BA) & $\begin{array}{c}\text { Apresentação em } \\
\text { Ciongresso }\end{array}$ & $\begin{array}{l}\text { Projeto de extensão interdisciplinar } \\
\text { como parte da formação pessoal e pro- } \\
\text { fissional de estudantes de Nutrição em } \\
\text { universidade pública brasileira. }\end{array}$ & $\begin{array}{l}\text { X Congresso de Nutrição e } \\
\text { Alimentação \& II Congresso } \\
\text { Ibero-Americano de Nutri- } \\
\text { ção, 2OII, Lisboa. }\end{array}$ \\
\hline
\end{tabular}

